\title{
Application of Normalized Compression Distance and Lempel-Ziv Jaccard Distance in Micro-electrode Signal Stream Classification for the Surgical Treatment of Parkinson's Disease
}

\section{Kamil Ząbkiewicz ${ }^{1}$}

${ }^{1}$ Faculty of Economics and Informatics in Vilnius, University of Bialystok, Vilnius, Lithuania

\begin{abstract}
Parkinson's Disease can be treated with the use of microelectrode recording and stimulation. This paper presents a data stream classifier that analyses raw data from micro-electrodes and decides whether the measurements were taken from the subthalamic nucleus (STN) or not. The novelty of the proposed approach is based on the fact that distances based on raw data are used. Two distances are investigated in this paper, i.e. Normalized Compression Distance (NCD) and Lempel-Ziv Jaccard Distance (LZJD). No new features needed to be extracted due to the fact that in the case of high-dimensional data the process is extremely time-consuming. The k-nearest neighbour (k-NN) was chosen as the classifier due to its simplicity, which is essential in data stream classification. Results obtained from classifiers based on k-NN: k-NN, k-NN were compared with Probabilistic Approximate Window (k-NN with PAW); k-NN with Probabilistic Approximate Window and Adaptive Windowing (k-NN with PAW and ADWIN); and Self Adjusting Memory k-NN (SAM k-NN), which use the proposed distances, with the performance of the same classifiers but using standard Euclidean distance. Prequential accuracy was chosen as the performance measure. The results of the experiments performed with the described approach are in most cases better, i.e. the performance measures for $\mathrm{kNN}$ classifiers that use NCD and LZJD distances are better by up to 8.5 per cent and 14 per cent, respectively. Moreover, the proposed approach performs better when compared with other stream classification algorithms, i.e. Hoeffding Tree, Naive Bayes, and Leveraging Bagging. In the discussed case, an improvement of classification rate of up to 17.9 per cent when using Lempel-Ziv Jaccard Distance instead of the Euclidean was noted.
\end{abstract}

\section{Introduction}

Parkinson's Disease (PD) is the second most common neurodegenerative disorder, affecting $2-3 \%$ of the human population aged over 65 . The latest primer on the disease presents several efforts concerning treatment: 
- Dopaminergic pharmacological treatment

- Non-dopaminergic pharmacological treatment

- Deep Brain Stimulation (DBS)

- Exercise-based treatment (Poewe et al., 2017)

Parkinson's Disease can be treated with the use of microelectrode recording and stimulation. The technique is called deep brain stimulation (DBS). The target of the surgery is located in a structure called subthalamic nucleus (STN), deep within the brain. DBS is based on the finding that high-frequency $(100-200 \mathrm{~Hz})$ electrical stimulation of specific brain targets can mimic the effect of a lesion without the need for destroying the brain tissue, and involves the implantation of an electrode in the brain tissue. The STN can be roughly divided into three functional territories: sensorimotor, associative, and limbic. It is crucial that only the sensorimotor part of the STN should be stimulated. STN is poorly distinguishable from the adjacent brain tissue on computed tomography (CT) and magnetic resonance imaging (MRI) scans. Those scans, however, do show other brain features. Using their referencing coordinates, the expected location of the STN can be approximated. Having only an approximate location of the STN, a set $(3 \sim 5)$ of parallel microelectrodes is inserted into the patient's brain during DBS surgery. Those are thin supporting electrodes, not to be implanted into the patient's brain permanently. The electrodes are directed towards the expected location of the target nucleus. At each desired depth, the electrodes can record electrophysiological activity of the surrounding brain tissue. The recordings are typically made at depths ranging from $10 \mathrm{~mm}$ above to $5 \mathrm{~mm}$ below the estimated STN location. When the trajectory to the STN and its depth are obtained in this manner, all microelectrodes are withdrawn to its level. Then, those that reach it sufficiently are one by one briefly switched from recording to stimulation mode. While stimulation is active, the patient's condition is monitored and the effectiveness of treatment assessed by a neurologist. By means of such a procedure, optimal electrode and depth are determined. Finally, all microelectrodes are withdrawn and, using the trajectory of the optimal microelectrode, a permanent stimulating electrode is implanted. It is to remain within the patient's brain (Ciecierski, 2013). This paper proposes a new approach based on data stream classification and distances derived from data compression and hashing to distinguish whether electrodes hit the STN region or not.

The paper is organized as follows: first, the problem of research is described and the state of the art in the area of the use of machine learning methods in DBS is presented. Secondly, Normalized Compression and 
Lempel-Ziv Jaccard distances are described. Then, requirements for the classifier are formulated, with a description of the data used in the experiments, and details on how they were performed are given. Finally, the results are discussed, including conclusions and targets for future research.

\section{Machine Learning Approach Used in DBS}

DBS technique has been known for 30 years, and over the past few years a number of interdisciplinary works concerning the use of machine learning methods have appeared, extending its functionality.

Kuhner et al. (2017) used Random Forests with probability distributions to detect abnormal motor behaviour of PD patients performing several different motor tasks in two clinical conditions (DBS switch: off, on). Trevathan et al. (2017) modelled dopamine release evoked by electrical stimulation using Artificial Neural Networks and Volterra kernels. Mamun et al. (2015) performed feature extraction and subset selection from local field potential signals and used Bayesian and Support Vector Machines (SVM) classifiers to decode movements. Rajpurohit et al. (2015) used a wrapper approach (by using a classifier) to select the features needed for further STN detection. Taghva (2011) used Hidden Semi-Markov Models for the detection of STN sub-regions. Valsky et al. (2017) used Support Vector Machines and Hidden Markov models to detect STN borders during DBS and thus reduce inadequate clinical outcomes of the surgery. Wong et al. (2009) performed feature extraction and fuzzy clustering from recordings acquired during DBS surgery to form a neural activity map to localize STN. Teplitzky et al. (2016) proposed a technique that uses feature selection and classification algorithms to work with DBS arrays. Hebb et al. (2014), Santaniello et al. (2018), Hell et al. (2018), Mohammed et al. (2018), Su et al. (2018) provided reviews and models for using closed-loop systems in DBS to make it adaptive. O'Halloran et al. (2016) and Shamir et al. (2018) connect STN detection with neuroimaging. Shamir et al. (2015) proposed a clinical support decision system to provide effective stimulation and adequate drug dosages, based on three machine learning methods, which included Support Vector Machines, Naïve Bayes, and Random Forest.

Most of the reviewed techniques are based on feature selection. The procedure takes time to complete, especially when high-dimensional and dynamic data is dealt with. A good example of this kind of data are microelectrode measurements used in DBS. In the following sections, distances 
independent of the type of features, but dependent on their binary representation, are dealt with.

\section{Normalized Compression Distance}

Normalized Compression Distance (NCD) is an approximation of the uncomputable Kolmogorov complexity function. The main formula for computing this measure is:

$$
N C D(x, y)=\frac{C(x y)-\min \{C(x), C(y)\}}{\max \{C(x), C(y)\}}
$$

where $C($.$) is the number of bytes obtained after performing compression of$ a binary string, while $x y$ - is the concatenation of binary strings.

According to Cilibrasi and Vitanyi (2005), if the compressor is normal, then the NCD is a normalized admissible distance satisfying the metric (in)equalities.

Requirements for normal compressor:

1. Idempotency: $C(x x)=C(x)$, and $C(\lambda)=0$, where $\lambda$ is the empty string

2. Monotonicity: $C(x y) \geq C(x)$

3. Symmetry: $C(x y)=C(y x)$

4. Distributivity: $C(x y)+C(z) \leq C(x z)+C(y z)$

It is also worth noting that lossless data compression is used for NCD computing. This guarantees that all compressed information can be restored in the future if archiving it is planned. An overview of the practical application of this distance can be found in (Cilibrasi, 2007; Cohen \& Vitanyi, 2015). Its direct application to stream classification has not been found.

\section{Lempel-Ziv Jaccard Distance}

The main approach used in computing the measure in question, proposed by Raff and Nicholas (2017), is based on LZ77 algorithm, especially on its part generating a set of all the substrings of given binary strings. Having two sets of substrings, the Jaccard similarity index is computed.

$$
J(A, B)=\frac{|A \cap B|}{|A \cup B|}
$$


Then, it is subtracted from 1 to obtain Lempel-Ziv Jaccard Distance (LZJD). This theoretical approach, according to its authors, was not as fast as desired, so they proposed an approximation based on hashing. It exploits the fact that the Jaccard similarity index can be computed approximately from a smaller digest produced from the original sets using a hashing function.

The formula for computing the approximation is as follows:

$$
J(A, B) \approx J\left(\bigcup_{j=1}^{k} h_{\min }^{j}(A), \bigcup_{j=1}^{k} h_{\min }^{j}(B)\right)
$$

where $h_{\text {min }}^{n}(S)$ indicates the $n$ 'th smallest hash values from set $S$.

The proposed measure was successfully used in malware classification. Literature review shows that it has not been used in data stream classification.

\section{Experiment Design}

All the experiments were performed in a Massive Online Analysis (MOA) environment. This application is written in Java and is one of the most popular solutions for data stream analysis. More detailed information concerning the use of MOA can be found in (Bifet et al., 2018).

The reason for choosing the k-nearest neighbour (k-NN) classifier is its simplicity and the minimal number of parameters. Moreover, it accepts not only numerical data. It is known that the algorithm relies on the distance measure that is used for finding the nearest neighbours and can be computed from numerical features, e.g. Euclidean or Mahalanobis distance. In addition, there are also distances that do not rely on numerical features. As examples, Normalized Compression Distance and LempelZiv Jaccard Distance can be given, which constitute the subject of this paper.

The performance of the approach proposed by the authors was compared with the performances of all k-NN classifier implementations used for data streams classification. Brief descriptions of the algorithms are provided below.

The k-NN classifier is the known algorithm, but in this case it is implemented to deal with streaming data. It contains the following parameters, which can be modified: 
- Number of neighbours: $k$ (default 10)

- Maximum number of instances to store: $w$ (default 1000)

- Nearest neighbour search method: $n$ (default LinearNN)

K-NN with Probabilistic Approximate Window (K-NN with PAW) is a modification of k-NN described in (Bifet et al., 2013). Probabilistic Approximate Window provides a compromise between relevance of information in the most recent instances and preservation of information carried by older instances. The parameters that can be modified are the same as in the standard k-NN.

K-NN with Probabilistic Approximate Window and Adaptive Windowing (K-NN with PAW and ADWIN) is another modification of $\mathrm{k}-\mathrm{NN}$ described in (Bifet et al., 2013). To enable adaptation to concept drift, the ADWIN change detector is added. In this case, the modifiable parameters are the same as in the case of $\mathrm{k}-\mathrm{NN}$.

Self-Adjusting Memory k-NN (SAM k-NN) is the newest modification of the k-NN classifier proposed in (Losing et al., 2018). It includes two memory types to deal with different types of concept drift, called shortterm memory (STM) and long-term memory (LTM). Several parameters of the algorithm can be modified:

- Number of neighbours: $k$ (default 5 )

- Maximum number of instances to store: $w$ (default 5000)

- Minimum number of instances in the STM: $m$ (default 50)

- Allowed LTM size relative to the total limit: $p$ (default 0.4)

- Recalculation of the error rate of the STM for size adaptation: $r$ (default is false, because of the computational cost)

The datasets used in the experiments was provided by Dr Eng. K. A. Ciecierski. He used it in his $\mathrm{PhD}$ thesis research. For the purpose of the experiments, records of three patients were used. The data was not preprocessed. Each record contained 240,000 measurements. Some records were not taken into account, due to the smaller number of measurements. The parameter that was changed during the experiments was the number of neighbours. Odd values between 1 and 9 were picked, while other variables required for the algorithms were set to their default values.

Moreover, the performance of the proposed approach was compared with other stream classifiers presented by Bifet et al. (2013), such as Hoeffding Tree, Naive Bayes, and Leveraging Bagging. The algorithms' parameters were set to their default values.

Prequential Accuracy was used as a classifier performance measure. It is defined in the following way: suppose we have a data stream. Each arriving instance can be used to test the model before it is used for training, and 
from this, the accuracy can be incrementally updated. As a result, the model is always being tested on instances that it has not seen before. Prequential Accuracy is computed at every moment $t$ as soon as prediction $\hat{y}_{t}$ is made.

$$
A c c(t)=\frac{1}{t} \sum_{i=1}^{t} L\left(y_{i}, \hat{y}_{i}\right)
$$

where $L\left(y_{i}, \hat{y}_{i}\right)=1$ if $y_{i}=\hat{y}_{i}$ and 0 if otherwise.

\section{Results and Discussion}

The results of the experiments performed with measurements in the first patient are shown in Table 1. Performance measures for the k-NN classifiers with NCD and LZJD are better by up to 8.5 per cent and 12.7 per cent, respectively. It can also be noticed that in order to obtain good results, the number of neighbours can be limited to only one.

Table 1. K-nearest neighbour classification using Euclidean distance, Normalized Compression Distance and Lempel-Ziv Jaccard Distance for the measurements taken from the first patient

\begin{tabular}{|c|c|c|c|c|c|c|c|c|}
\hline & \multicolumn{4}{|c|}{ k-NN_ED } & \multicolumn{4}{|c|}{ k-NN_NCD } \\
\hline NN & $\mathrm{k}-\mathrm{NN}$ & $\begin{array}{l}\text { k-NN+ } \\
\text { PAW }\end{array}$ & $\begin{array}{l}\text { k-NN+ } \\
\text { PAW+ } \\
\text { ADWIN }\end{array}$ & $\begin{array}{l}\text { SAM } \\
\text { k-NN }\end{array}$ & $\mathrm{k}-\mathrm{NN}$ & $\begin{array}{l}\text { k-NN+ } \\
\text { PAW }\end{array}$ & $\begin{array}{l}\text { k-NN+ } \\
\text { PAW+ } \\
\text { ADWIN }\end{array}$ & $\begin{array}{l}\text { SAM } \\
\text { k-NN }\end{array}$ \\
\hline 1 & 70.707 & 71.717 & 71.717 & 67.677 & \multirow{5}{*}{77.778} & \multirow{5}{*}{\multicolumn{2}{|c|}{73.737}} & 75.758 \\
\hline 3 & 71.717 & 71.717 & 71.717 & 65.657 & & & & 68.687 \\
\hline 5 & 71.717 & 73.737 & 73.737 & 68.687 & & & & 59.596 \\
\hline 7 & 68.687 & 72.727 & 72.727 & 65.657 & & & & 54.545 \\
\hline 9 & 57.575 & 72.727 & 72.727 & 53.535 & & & & 56.566 \\
\hline & \multicolumn{4}{|c|}{ k-NN_LZJD } & & & & \\
\hline NN & $\mathrm{k}-\mathrm{NN}$ & $\begin{array}{l}\text { k-NN+ } \\
\text { PAW }\end{array}$ & $\begin{array}{l}\text { k-NN+ } \\
\text { PAW+ } \\
\text { ADWIN }\end{array}$ & $\begin{array}{l}\text { SAM } \\
\text { k-NN }\end{array}$ & & & & \\
\hline 1 & \multirow{5}{*}{\multicolumn{3}{|c|}{80.808}} & 77.778 & & & & \\
\hline 3 & & & & 68.687 & & & & \\
\hline 5 & & & & 63.636 & & & & \\
\hline 7 & & & & 54.545 & & & & \\
\hline 9 & & & & 59.596 & & & & \\
\hline
\end{tabular}


Table 2. K-nearest neighbour classification using Euclidean distance, Normalized Compression Distance, and Lempel-Ziv Jaccard Distance for the measurements taken from the second patient

\begin{tabular}{|c|c|c|c|c|c|c|c|c|}
\hline & \multicolumn{4}{|c|}{ k-NN_ED } & \multicolumn{4}{|c|}{$\mathrm{k}-\mathrm{NN} \_\mathrm{NCD}$} \\
\hline NN & $\mathrm{k}-\mathrm{NN}$ & $\begin{array}{l}\text { k-NN+ } \\
\text { PAW }\end{array}$ & $\begin{array}{l}\text { k-NN+ } \\
\text { PAW+ } \\
\text { ADWIN }\end{array}$ & $\begin{array}{l}\text { SAM } \\
\text { k-NN }\end{array}$ & $\mathrm{k}-\mathrm{NN}$ & $\begin{array}{l}\text { k-NN+ } \\
\text { PAW }\end{array}$ & $\begin{array}{l}\text { k-NN+ } \\
\text { PAW+ } \\
\text { ADWIN }\end{array}$ & $\begin{array}{l}\text { SAM } \\
\text { k-NN }\end{array}$ \\
\hline 1 & 71.605 & 72.840 & 72.840 & 66.667 & \multirow{5}{*}{75.309} & \multirow{5}{*}{\multicolumn{2}{|c|}{76.543}} & 75.309 \\
\hline 3 & 71.605 & 71.605 & 71.605 & 69.136 & & & & 70.370 \\
\hline 5 & 71.605 & 70.370 & 70.370 & 66.667 & & & & 58.025 \\
\hline 7 & 71.605 & 67.901 & 67.901 & 61.728 & & & & 56.790 \\
\hline 9 & 70.370 & 67.901 & 67.901 & 59.259 & & & & 55.556 \\
\hline & \multicolumn{4}{|c|}{ k-NN_LZJD } & & & & \\
\hline NN & $\mathrm{k}-\mathrm{NN}$ & $\begin{array}{l}\text { k-NN+ } \\
\text { PAW }\end{array}$ & $\begin{array}{l}\text { k-NN+ } \\
\text { PAW+ } \\
\text { ADWIN }\end{array}$ & $\begin{array}{l}\text { SAM } \\
\text { k-NN }\end{array}$ & & & & \\
\hline 1 & \multirow{5}{*}{79.012} & \multirow{5}{*}{\multicolumn{2}{|c|}{81.481}} & 76.543 & & & & \\
\hline 3 & & & & 70.370 & & & & \\
\hline 5 & & & & 65.432 & & & & \\
\hline 7 & & & & 60.494 & & & & \\
\hline 9 & & & & 58.025 & & & & \\
\hline
\end{tabular}

The classification results for the data from the second patient are shown in Table 2. Performance measures for the k-NN classifiers with NCD and LZJD distances are better by up to 7 per cent and 14 per cent, respectively. Limitation of the number of neighbours to only one also tends to give good results.

The classification results for the data from the third patient are shown in Table 3. In this case, it can be noticed that the approach that uses LZJD performs the best. The performance is better by 5 per cent in comparison with the case where standard Euclidean distance is used. In most cases, the k-NN classifier variants that use NCD perform similarly to the k-NN classifier variants with Euclidean distance. Similarly to previous cases, the number of neighbours can be limited to only one.

The results of classification performance of the other stream classifiers are provided in Table 4 . When compared with the k-NN mentioned earlier, the variants with NCD and LZJD, it can be seen that the performance overcomes the performance of the Hoeffding Tree, Naive Bayes, and Leveraging 
Application of Normalized Compression Distance and Lempel-Ziv Jaccard...

Table 3. K-nearest neighbour classification using Euclidean distance, Normalized Compression Distance, and Lempel-Ziv Jaccard Distance for the measurements taken from the third patient

\begin{tabular}{|c|c|c|c|c|c|c|c|c|}
\hline & \multicolumn{4}{|c|}{ k-NN_ED } & \multicolumn{4}{|c|}{ k-NN_NCD } \\
\hline NN & k-NN & $\begin{array}{l}\text { k-NN+ } \\
\text { PAW }\end{array}$ & $\begin{array}{l}\text { k-NN+ } \\
\text { PAW+ } \\
\text { ADWIN }\end{array}$ & $\begin{array}{l}\text { SAM } \\
\text { k-NN }\end{array}$ & $\mathrm{k}-\mathrm{NN}$ & $\begin{array}{l}\text { k-NN+ } \\
\text { PAW }\end{array}$ & $\begin{array}{l}\text { k-NN+ } \\
\text { PAW+ } \\
\text { ADWIN }\end{array}$ & $\begin{array}{l}\text { SAM } \\
\text { k-NN }\end{array}$ \\
\hline 1 & \multirow{5}{*}{\multicolumn{3}{|c|}{89.247}} & 88.172 & \multirow{5}{*}{88.172} & \multirow{5}{*}{\multicolumn{2}{|c|}{89.247}} & 87.097 \\
\hline 3 & & & & 88.172 & & & & 79.570 \\
\hline 5 & & & & 89.247 & & & & 81.720 \\
\hline 7 & & & & 88.172 & & & & 84.946 \\
\hline 9 & & & & 86.022 & & & & 81.720 \\
\hline & \multicolumn{4}{|c|}{ k-NN_LZJD } & & & & \\
\hline NN & $\mathrm{k}-\mathrm{NN}$ & $\begin{array}{l}\text { k-NN+ } \\
\text { PAW }\end{array}$ & $\begin{array}{l}\text { k-NN+ } \\
\text { PAW+ } \\
\text { ADWIN }\end{array}$ & $\begin{array}{l}\text { SAM } \\
\text { k-NN }\end{array}$ & & & & \\
\hline 1 & \multirow{5}{*}{91.398} & \multirow{5}{*}{\multicolumn{2}{|c|}{93.548}} & 91.398 & & & & \\
\hline 3 & & & & 91.398 & & & & \\
\hline 5 & & & & 84.946 & & & & \\
\hline 7 & & & & 86.022 & & & & \\
\hline 9 & & & & 86.022 & & & & \\
\hline
\end{tabular}

Table 4. Classification results obtained for three patients with the use of other popular stream classifiers

\begin{tabular}{|c|c|c|c|c|c|}
\hline $\begin{array}{c}\text { Number of } \\
\text { a patient }\end{array}$ & $\begin{array}{c}\text { Naive } \\
\text { Bayes }\end{array}$ & $\begin{array}{c}\text { Hoeffding } \\
\text { Tree }\end{array}$ & $\begin{array}{c}\text { Leveraging } \\
\text { Bagging }\end{array}$ & $\begin{array}{c}\text { k-NN } \\
\text { NCD }\end{array}$ & $\begin{array}{c}\text { k-NN } \\
\text { LZJD }\end{array}$ \\
\hline 1 & 71.717 & 71.717 & 70.707 & 77.778 & 80.808 \\
\hline 2 & 71.605 & 71.605 & 69.136 & 76.543 & 81.481 \\
\hline 3 & 89.247 & 89.247 & 89.247 & 89.247 & 93.548 \\
\hline
\end{tabular}

Bagging in the case of all three patients, or tends to be the same. The improvement of the performance rate for the first patient is an up to 10 per cent increase in the case of $\mathrm{k}-\mathrm{NN}$ with $\mathrm{NCD}$ and up to 14.3 per cent for LZJD. In the case of the second patient, an improvement of up to 10.7 and 17.9 per cent can be seen, respectively. Finally, when classifying the data from the third patient, improvement of up to 4.8 per cent is achieved when using LZJD distance. 
To sum up, classification rates achieved with the proposed measures are in most cases better than with the standard (Euclidean) distance. It is also worth noticing that in most cases the authors' approach gives better results than other data stream classification algorithms. As mentioned before, computations were made on non-filtered/preprocessed numerical data.

\section{Conclusions}

The experimental results showed the usability of the proposed distance measures in classifying high-dimensional medical measurement streaming data. The experiments were performed on raw, noisy (in most cases) data. In future research, signal denoising is planned to be performed by cutting off unnecessary frequencies and filter the signal with high-pass and low-pass filters. The structure of the data will remain unchanged, i.e. all the 240,000 measurements will be available. No new derivative features will be extracted from the data. Then, experiments such as those described in this paper will be performed and their results will be compared. Another approach to obtain better results is to perform Prequential Cross-validation. Future experiments will test this assumption. One of the issues that occurred during the experiments is longer computation time, especially in the case of the Normalized Compression Distance. Obviously, several causes can be found. First of all, code written in Java works slower than its implementation in C language (many compression algorithms are implemented when using it). Secondly, when the k-nearest neighbour classification is performed, distances are each time computed from the beginning. For this reason, some caching of known distances could result in the algorithm's faster work. Finally, parallelization was not used. Hence, splitting the compression task for simultaneous threads could potentially result in a speedup. Advantage can also be taken of the fact that when using the proposed distances, the number of nearest neighbours can be limited to only one, which would provide a good classification rate.

Nonetheless, the results look promising and after some improvement to the implementation, it may become a useful tool that will help to perform deep brain stimulation more precisely.

\section{Acknowledgements}

Computations performed as part of the experiments were carried out at the Computer Center of the University of Bialystok. We also wish to thank Dr Eng. Konrad A. Ciecierski for providing the data sets for the experiments. 
Bifet, A., Pfahringer, B., Read, J., \& Holmes, G. (2013). Efficient Data Stream Classification via Probabilistic Adaptive Windows. In Proceedings of the 28th Annual ACM Symposium on Applied Computing (pp. 801-806). New York, NY, USA: ACM. doi: 10.1145/2480362.2480516

Bifet, A., Gavaldà, R., Holmes, G., \& Pfahringer, B. (2018). Machine Learning for Data Streams with Practical Examples in MOA. MIT Press.

Ciecierski, K. A. (2013). Decision Support System for surgical treatment of Parkinson's disease (PhD thesis). Warsaw University of Technology.

Cilibrasi, R., \& Vitanyi, P. M. B. (2005). Clustering by compression. IEEE Transactions on Information Theory, 51(4), 1523-1545. doi: 10.1109/TIT.2005. 844059

Cilibrasi, R. (2007). Statistical Inference Through Data Compression (PhD thesis). Institute for Logic, Language and Computation, University of Amsterdam.

Cohen, A. R., \& Vitanyi, P. M. B. (2015). Normalized Compression Distance of Multisets with Applications. IEEE Transactions on Pattern Analysis and Machine Intelligence, 37(8), 1602-1614. doi: 10.1109/TPAMI.2014.2375175

Hebb, A. O., Zhang, J. J., Mahoor, M. H., Tsiokos, C., Matlack, C., Chizeck, H. J., \& Pouratian, N. (2014). Creating the Feedback Loop. Neurosurgery Clinics of North America, 25(1), 187-204. doi: 10.1016/j.nec.2013.08.006

Hell, F., Köglsperger, T., Mehrkens, J., \& Boetzel, K. (2018). Improving the Standard for Deep Brain Stimulation Therapy: Target Structures and Feedback Signals for Adaptive Stimulation. Current Perspectives and Future Directions. Cureus, 10(4). doi: 10.7759/cureus.2468

Kuhner, A., Schubert, T., Cenciarini, M., Wiesmeier, I. K., Coenen, V. A., Burgard, W., Weiller, C., et al. (2017). Correlations between Motor Symptoms across Different Motor Tasks, Quantified via Random Forest Feature Classification in Parkinson's Disease. Frontiers in Neurology, 8:607. doi: 10.3389/fneur.2017.00607

Losing, V., Hammer, B., \& Wersing, H. (2018). Tackling heterogeneous concept drift with the Self-Adjusting Memory (SAM). Knowledge and Information Systems, 54(1), 171-201. doi: 10.1007/s10115-017-1137-y

Mamun, K. A., Mace, M., Lutman, M. E., Stein, J., Liu, X., Aziz, T., Vaidyanathan, R., et al. (2015). Movement decoding using neural synchronization and inter-hemispheric connectivity from deep brain local field potentials. Journal of Neural Engineering, 12(5), 056011. doi: 10.1088/1741-2560/12/5/056011

Mohammed, A., Bayford, R., \& Demosthenous, A. (2018). Toward adaptive deep brain stimulation in Parkinson's disease: a review. Neurodegenerative Disease Management, 8(2), 115-136. doi: 10.2217/nmt-2017-0050

O'Halloran, R., Kopell, B. H., Sprooten, E., Goodman, W. K., \& Frangou, S. (2016). Multimodal Neuroimaging-Informed Clinical Applications in Neuropsychiatric Disorders. Frontiers in Psychiatry, 7:63. doi: 10.3389/fpsyt.2016.00063 
Poewe, W., Seppi, K., Tanner, C. M., Halliday, G. M., Brundin, P., Volkmann, J., Schrag, A. L., et al. (2017). Parkinson disease. Nature Reviews Disease Primers, 3:17013. doi: 10.1038/nrdp.2017.13

Raff, E., \& Nicholas, C. (2017). An Alternative to NCD for Large Sequences, Lempel-Ziv Jaccard Distance. In Proceedings of the 23rd ACM SIGKDD International Conference on Knowledge Discovery and Data Mining (pp. 10071015). ACM Press. doi: 10.1145/3097983.3098111

Rajpurohit, V., Danish, S. F., Hargreaves, E. L., \& Wong, S. (2015). Optimizing computational feature sets for subthalamic nucleus localization in DBS surgery with feature selection. Clinical Neurophysiology, 126(5), 975-982. doi: $10.1016 /$ j.clinph.2014.05.039

Santaniello, S., Gale, J. T., \& Sarma, S. V. (2018). Systems approaches to optimizing deep brain stimulation therapies in Parkinson's disease. Wiley Interdisciplinary Reviews: Systems Biology and Medicine, 10(5), e1421. doi: $10.1002 / w s b m .1421$

Shamir, R. R., Dolber, T., Noecker, A. M., Walter, B. L., \& McIntyre, C. C. (2015). Machine Learning Approach to Optimizing Combined Stimulation and Medication Therapies for Parkinson's Disease. Brain Stimulation, 8(6), 1025-1032. doi: 10.1016/j.brs.2015.06.003

Shamir, R. R., Duchin, Y., Kim, J., Patriat, R., Marmor, O., Bergman, H., Vitek, J. L., et al. (2018). Microelectrode Recordings Validate the Clinical Visualization of Subthalamic-Nucleus Based on 7T Magnetic Resonance Imaging and Machine Learning for Deep Brain Stimulation Surgery. Neurosurgery. doi: 10.1093/neuros/nyy 212

Su, F., Wang, J., Niu, S., Li, H., Deng, B., Liu, C., \& Wei, X. (2018). Nonlinear predictive control for adaptive adjustments of deep brain stimulation parameters in basal ganglia-thalamic network. Neural Networks, 98, 283-295. doi: 10.1016/j.neunet.2017.12.001

Taghva, A. (2011). Hidden Semi-Markov Models in the Computerized Decoding of Microelectrode Recording Data for Deep Brain Stimulator Placement. World Neurosurgery, 75(5-6), 758-763.e4. doi: 10.1016/j.wneu.2010.11.008

Teplitzky, B. A., Zitella, L. M., Xiao, Y., \& Johnson, M. D. (2016). Model-Based Comparison of Deep Brain Stimulation Array Functionality with Varying Number of Radial Electrodes and Machine Learning Feature Sets. Frontiers in Computational Neuroscience, 10:58. doi: 10.3389/fncom.2016.00058

Trevathan, J. K., Yousefi, A., Park, H. O., Bartoletta, J. J., Ludwig, K. A., Lee, K. H., \& Lujan, J. L. (2017). Computational Modeling of Neurotransmitter Release Evoked by Electrical Stimulation: Nonlinear Approaches to Predicting Stimulation-Evoked Dopamine Release. ACS Chemical Neuroscience, 8(2), 394-410. doi: 10.1021/acschemneuro.6b00319

Valsky, D., Marmor-Levin, O., Deffains, M., Eitan, R., Blackwell, K., Bergman, H., \& Israel, Z. (2017). Stop! Border Ahead: Automatic detection of subthalamic 
Application of Normalized Compression Distance and Lempel-Ziv Jaccard...

exit during deep brain stimulation surgery. Movement Disorders, 32(1), 70 79. doi: $10.1002 /$ mds. 26806

Wong, S., Baltuch, G. H., Jaggi, J. L., \& Danish, S. F. (2009). Functional localization and visualization of the subthalamic nucleus from microelectrode recordings acquired during DBS surgery with unsupervised machine learning. Journal of Neural Engineering, 6(2), 026006. doi: 10.1088/1741-2560/6/2/026006 